\title{
Reducing the barriers against analytical epidemiological studies in investigations of local foodborne disease outbreaks in Germany - a starter kit for local health authorities
}

D Werber (werberd@rki.de) ${ }^{1}$, H Bernard ${ }^{1}$

1. Department of Infectious Disease Epidemiology, Robert Koch-Institut, Berlin, Germany

Citation style for this article:

Werber D, Bernard H. Reducing the barriers against analytical epidemiological studies in investigations of local foodborne disease outbreaks in Germany - a starter kit for local health authorities. Euro Surveill. 2014;19(8):pii=20714. Available online: http://www.eurosurveillance.org/ViewArticle.aspx?Articleld=20714

Article submitted on 22 February 2013 / published on 27 February 2014

Thousands of infectious food-borne disease outbreaks (FBDO) are reported annually to the European Food Safety Authority within the framework of the zoonoses Directive (2003/99/EC). Most recognised FBDO occur locally following point source exposure, but only few are investigated using analytical epidemiological studies. In Germany, and probably also in other countries of the European Union, this seems to be particularly true for those investigated by local health authorities. Analytical studies, usually cohort studies or casecontrol studies, are a powerful tool to identify suspect food vehicles. Therefore, from a public health and food safety perspective, their more frequent usage is highly desirable. We have developed a small toolbox consisting of a strategic concept and a simple software tool for data entry and analysis, with the objective to increase the use of analytical studies in the investigation of local point source FBDO in Germany.

\section{Introduction}

Outbreak identification, investigation, and control are primary objectives for public health. The Directive $2003 / 99 / E C$ on the monitoring of zoonoses and zoonotic agents of the European Parliament and of the European Council defines food-borne disease outbreaks (FBDO) as two or more human cases of the same disease and/ or infection [...] and where the cases are linked, or are probably linked, to the same food source. FBDO, usually caused by infectious agents, occur frequently in Europe [1]. In 2010, for example, a total of 4,858 FBDO affecting 58,083 persons (data excluding those of Spain) were reported to the European Food Safety Authority (EFSA) [2]. Their effective control depends on the rapid identification of the suspected food vehicle from all the available epidemiological, microbiological, environmental and other evidence and on preventing its further consumption, e.g. by removing it from the market. From a food safety perspective, it is paramount to learn what went wrong during production and preparation of the food so that measures to prevent further outbreaks or sporadic cases can be implemented [3]. Most FBDO are recognised and handled locally [4], emphasising the necessity for local health authorities to be able to conduct timely epidemiological investigations, which can (but do not always have to) include analytical epidemiological studies, usually cohort studies or casecontrol studies. Importantly, these studies increase the likelihood of successfully identifying the suspected food vehicle. [4]

However, analytical epidemiological studies are seldom employed in the investigation of FBDO, particularly in local outbreaks, where control measures are often prompted by descriptive epidemiology and prior knowledge, i.e. biological plausibility, of common food vehicles. In 528 of 4,858 (11\%) FBDO reported to EFSA for 2010 , the evidence for a suspected food vehicle was reported as being 'strong', i.e. usually based on better evidence than a suspected vehicle's biological plausibility and on the fact that most or all of the cases had been exposed. Only in 148 of them (27\%; $3 \%$ of all reported $\mathrm{FBDO}$ ) had an analytical epidemiological study been conducted. The use of analytical epidemiology varies widely across the Member States of the European Union (EU), and some countries seemingly do not use them at all [2]. In 2010 in Germany, reports on 439 FBDO with an identified causative agent were electronically submitted to the public health institute at national level, the Robert Koch Institute (RKI). In 40 of these 439 (9\%), the evidence implicating a suspect food vehicle or meal was considered to be strong, but only in two of the 40 (i.e. $5 \%$ of those with strong evidence, and less than $1 \%$ of the total) had an analytical epidemiological study been reported, one by the state health department and one by a field epidemiologist trainee [5], both assisting local health authorities.

Obviously, barriers against employing analytical epidemiological studies in FBDO investigations exist. The reasons why analytical epidemiological studies in Germany are seldom employed are many and include lack of human or technical resources, conflicting public 
health priorities, late outbreak detection (resulting in no new cases at the start of a possible investigation), and lack of experience in conducting such studies. In our experience, the greatest barriers are associated with three core activities in epidemiological outbreak investigations:

1. Use of long hypothesis-generating (trawling) questionnaires,

2. Use of specific, sometimes complex software for data entry and/or statistical analysis,

3. Identifying and interviewing healthy persons serving as reference population (in cohort studies or case-control studies).

All these activities are usually not used outside of outbreak investigations by local health authorities in
Germany. Consequently, they require special resources, and some also require specific skills (points 2 and 3 ). To support local public health authorities in overcoming some of these barriers (namely points 1 and 2), we have developed a toolbox that includes a strategic concept and an electronic file (Linelist Tool) that allows for data entry and automatic analysis. We thereby hope to increase the use of analytical epidemiological comparisons in investigations of FBDO in Germany.

\section{The concept}

The concept of how to investigate epidemiologically a FBDO is based on the steps of an outbreak investigation [6] as taught in many field epidemiology training programmes around the world (e.g. the European Programme for Intervention Epidemiology Training (EPIET) or the Epidemic Intelligence Service (EIS) of

\section{FIGURE 1}

Simplified schematic for epidemiological investigation of foodborne disease outbreaks in Germany

(1) Verify outbreak, (2) confirm diagnosis, (3) define case, (4) (active) case finding
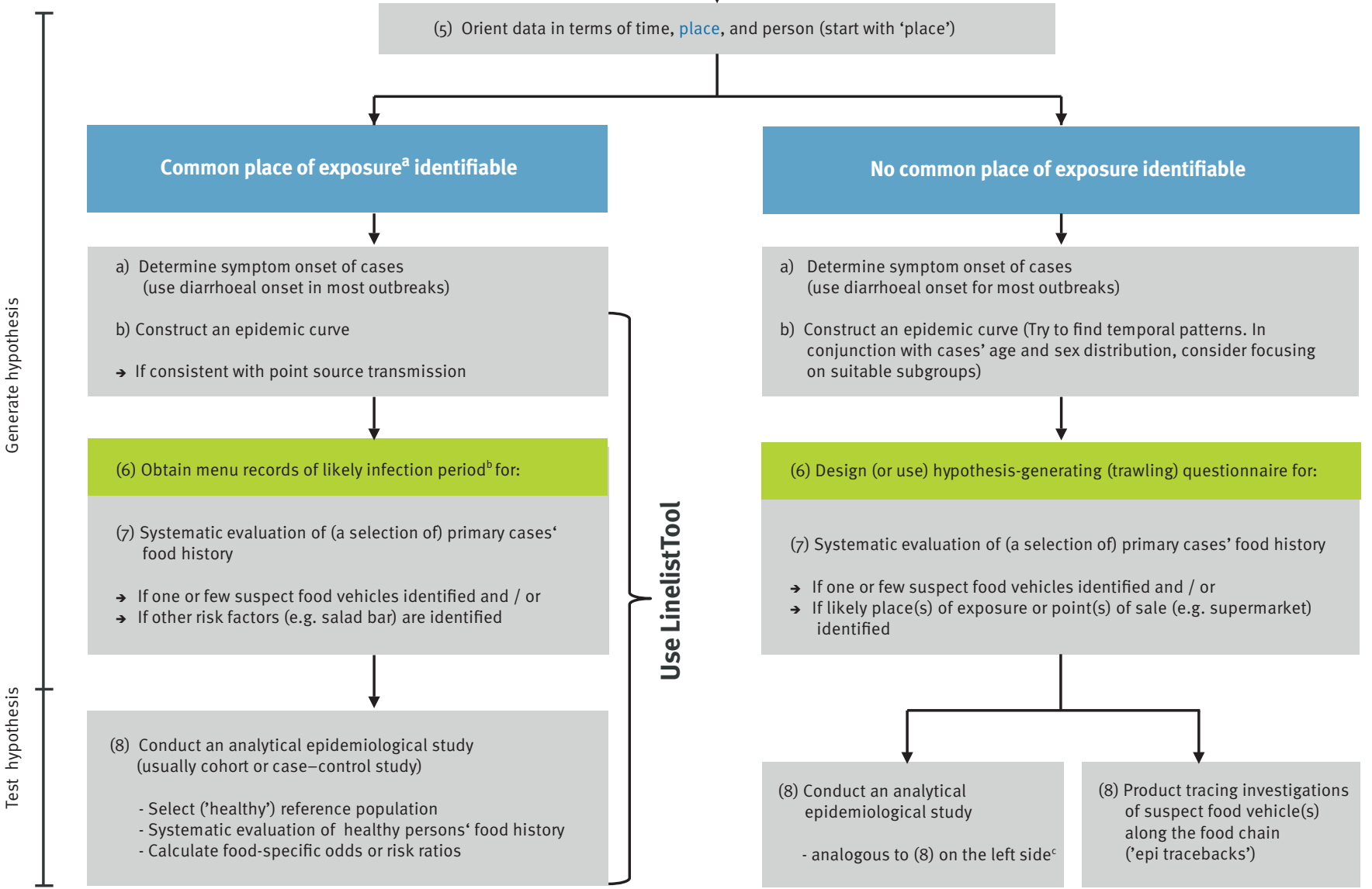

FBDO: foodborne disease outbreaks.

a Usually an institution, a restaurant or a social gathering.

b Infection period can be estimated for group of primary cases using the incubation period of the causal agent (if known), or those of typical FBDO agents.

Additionally or even alternatively, the association of specific food items with illness may be rapidly assessed using binomial probability, provided that estimates on the prevalence of consumption of these food items in the general population are available or that their range can be reasonably guessed [9]. 
the United States). The concept has been adapted primarily to distinguish outbreaks where a common local place of exposure can be identified, e.g. a social gathering or an institution, and outbreaks where this is not the case. The concept is displayed in Figure 1. FBDO come in various shapes and sizes, and thus the concept is oversimplifying (e.g. continuing common source outbreaks do occur in institutions, and the concept does not accommodate for them). By experience and for didactic purposes, it is assumed that most local outbreaks are recognised by a sudden increase in case numbers, and so the epidemic curve is suggestive of a point source transmission, indicating a single exposure event.

The salient point is that in these outbreaks, menu records can often be used instead of long trawling questionnaires as the basis for hypothesis-generating interviews with cases. We advise local health authorities to focus primarily on investigating local outbreaks with a point source exposure. The Linelist Tool should offer them assistance in their analytical epidemiological investigation. In the rarer outbreaks where cases are geographically scattered, we advise them to seek assistance from public health authorities at the federal state or the national level, as these investigations are usually more time-consuming, complex and often not restricted to one jurisdiction.

\section{The Linelist Tool}

The Linelist Tool is a Microsoft Excel (Microsoft Corporation, Redmond, Washington) file consisting of several spreadsheets. The first spreadsheet (Linelist) allows for the entry of data collected from cases and healthy control subjects (Figure 2).

The other spreadsheets are completed automatically, based on the data entered. In one spreadsheet, an epidemic curve of the outbreak is generated. This should help local investigators to determine the time period when exposure is likely to have occurred, and to identify cases that are likely to be primary cases, i.e. infected by a common source, to seek food histories from (a subset of) them. Other spreadsheets display univariable measures of association for each dichotomous exposure, i.e. food vehicle-specific associations, expressed as odds ratios or risk ratios, depending on whether a cohort or case-control design is used (only responses coded as 'yes' or 'no' are included in the analysis). In addition to these estimates, corresponding approximate $95 \%$ confidence intervals [7] indicate the precision of the estimation and can be used to assess statistical significance (Figure 3 ).

We offer a short training course for local health authorities which we recommend users to complete before applying the tool in an outbreak investigation. The Linelist Tool is currently available only in German

\section{FIGURE 2}

Linelist spreadsheet for entry of data collected on case-patients and control subjects in investigations of foodborne disease outbreaks in Germany

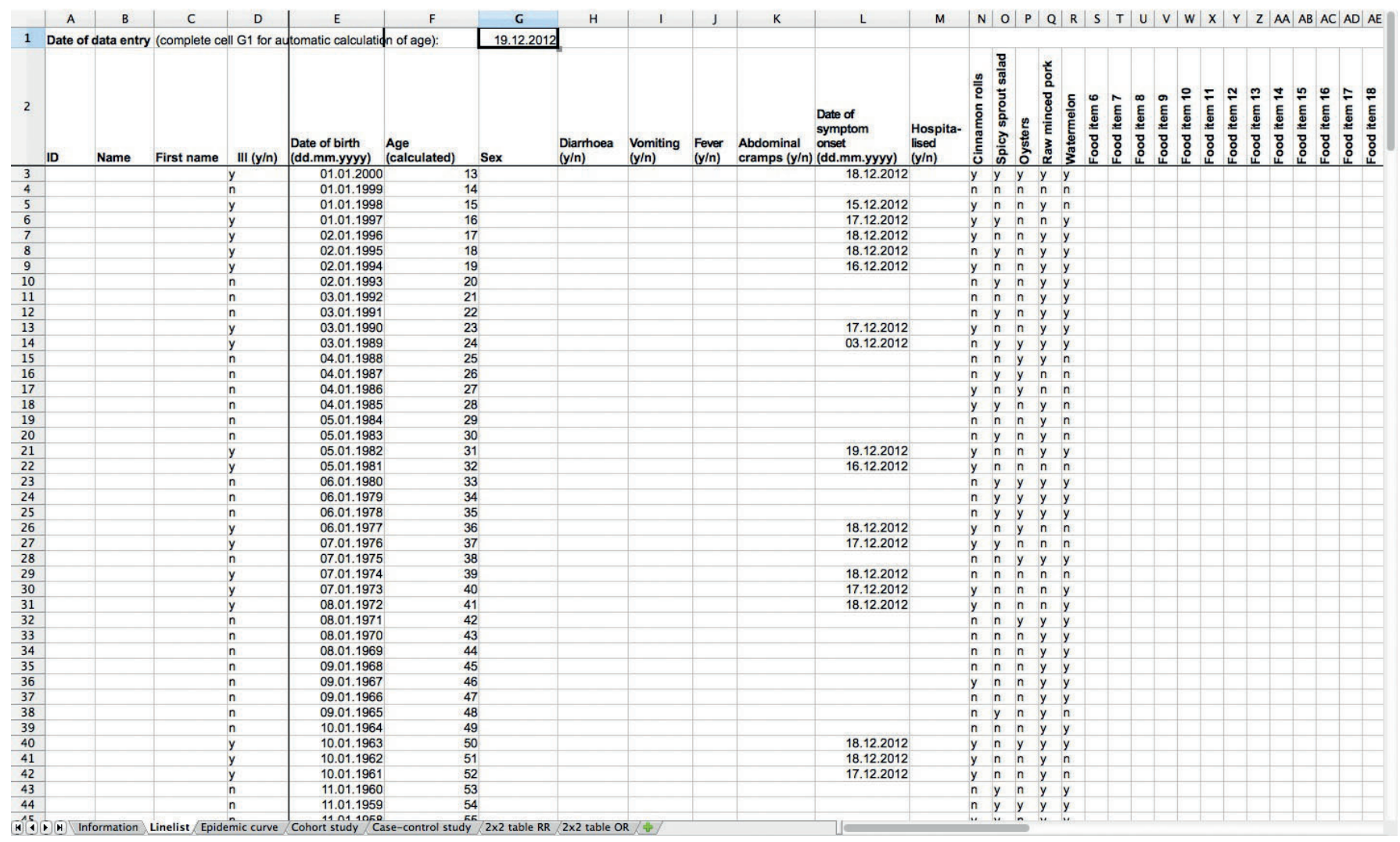


language and can be downloaded at http://www.rki. de/linelisttool. Planned future developments of the tool include a power table for unmatched case-control studies (based on case numbers and the proportion of cases exposed to the suspected vehicle) to inform local health authorities of an appropriate number of control subjects when deciding whether or not to conduct a case-control study.

\section{Discussion}

Epidemiological investigations of FBDO, if conducted, seldom go beyond the evaluation of cases' food histories. If the prevalence of consumption of a food in the general population can be assumed to be low (e.g. raw milk in Germany) or is indicated by background data such as consumption surveys (e.g. water cress in the UK [8]), this may provide sufficient descriptive epidemiological evidence to implicate a specific food vehicle, and more targeted investigations (e.g. food sampling) or control measures can be initiated. In these instances, delaying intervention measures because an analytical study has not been conducted or yet completed may put the public at unacceptable risk. More often than not, however, it remains unclear whether a food item frequently consumed by cases implicates the contaminated vehicle or merely reflects the popularity of the food in the source population. For illustration, in Germany in 2010, in 252 of the 399 $(63 \%)$ FBDO reported with 'weak' evidence, the only reported evidence implicating a food or meal was that 'the majority of cases consumed the implicated food' (a field entry in the electronic outbreak report). Probably most investigations ended inconclusively, no intervention occurred, and nothing could be learnt to inform improvements in food safety. To make matters worse, in some FBDO more than one food may be found to have been frequently consumed by cases. Irrespective of whether there are one or more such vehicles, an analytical epidemiological comparison is necessary to distinguish between popular foods and those associated with being a case.

We have developed a simple data entry and analysis tool for basic epidemiological comparisons in the investigation of point source FBDO. The main advantages of the tool are speed and ease of use. It only requires standard office software and the file is small enough to run on most computers. It does not require knowledge of specific statistical software (e.g. R, SAS, SPSS, Stata), but data can easily be imported by such software if more sophisticated analyses are needed. The software tool has already proven useful in a large norovirus outbreak linked to contaminated frozen strawberries [10], for which studies were completed, including analysis, within one day. In addition to FBDO, the tool can be used for the investigation of other outbreaks, e.g. of vaccine-preventable diseases. However, the tool may not be as suitable for the investigation

\section{FIGURE 3}

Spreadsheet 'Cohort study' with 2x2 tables displaying univariable measures of association for each dichotomous exposure in investigations of foodborne disease outbreaks in Germany

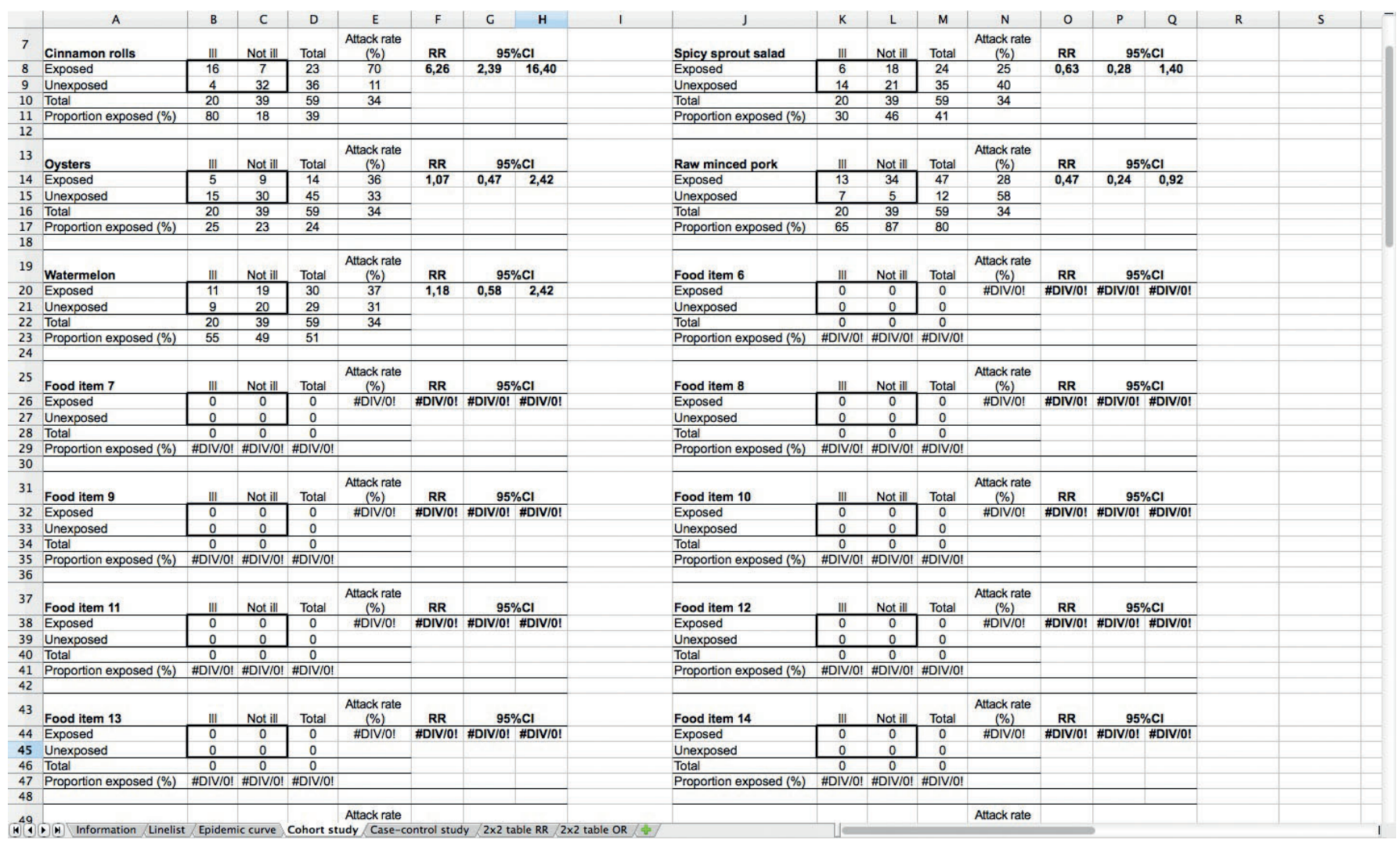


of geographically diffuse outbreaks where exposure lists are not readily available and where investigators initially rely on hypothesis-generating interviews with cases to identify exposures of interest that can be further investigated by an analytical study. Most reported FBDO in Europe with strong evidence for a food vehicle involve only a single place of exposure, implying that most of them are investigated by local health authorities. Furthermore, analytical epidemiological studies are seldom conducted in FBDO in Europe, considering the large number of FBDO reported to EFSA. We therefore believe that this starter kit may be useful in other countries of the EU and welcome suggestions and feedback for its further improvement.

As much as we are advocating the use of a simple analysis tool in investigations of local point source FBDO, we advise judicious use and data interpretation, and emphasise the importance of considering analytical epidemiological evidence in the context of all other available evidence, e.g. microbiological evidence and product-tracing evidence. It is important to note that the tool has purposely been kept simple and permits only univariable comparisons. The results are a starting point for further, more targeted, investigations to identify the ingredient of the implicated meal carrying the causal agent. These investigations could include more detailed statistical analysis, further analytical studies, additional food histories, and environmental investigations, as well as food sampling and product tracing. At least some of these investigations are necessary to identify a suspected vehicle and where and when contamination with (or multiplication of) the causative agent occurred.

We acknowledge that analytical epidemiological studies are not a universal remedy, and not all FBDO lend themselves to an analytical investigation. For example, some FBDO are small, which hampers meaningful studies. Among them are those that occur in a single household (often ascertained in the German surveillance system). It is difficult to provide a numeric threshold for when an analytical study should be conducted because the decision depends on many factors, such as the dynamic of the outbreak or severity of illnesses. Nevertheless, it is instructive to note that in 2010, 23 FBDO were reported in Germany, each with at least 20 human cases, but evidence from an analytical study had been reported for only one. This number of outbreaks is likely to be an underestimate because a causative agent is not always identified in FBDO. In such cases, information on the outbreaks is often not transmitted from the local level via the state health department to the RKI. The fact that a causative agent has not (yet) be identified in a FBDO should not hinder the use of the tool; clinical case definitions may be perfectly adequate alternatives to microbiological confirmation [10,11]. Epidemiologically identifying a suspected vehicle enables better targeted searches for the causative agent in food samples. In addition, it may allow estimating the incubation period, which may provide crucial hints at the aetiological agent, thereby informing microbiologic investigation of human cases. Again, the norovirus outbreak linked to frozen strawberries is an excellent example [10].

Investigation of FBDO requires adequate resources, which is challenging in times where budget deficits have already left their footprint on public health surveillance in many countries. However, surveillance of FBDO is necessary for understanding the epidemiology of foodborne diseases [4] and to serve the development of public health policies on food safety $[12,13]$ in a systematic and more unbiased fashion than focussing on published outbreaks [14]. The higher the proportion of FBDO with credibly identified food vehicles, the more valid is the evidence base provided by them.

We hope that by providing a simple software tool and by guiding local health authorities on how and which FBDO to investigate, more analytical studies in FBDO investigations will be conducted, and as a consequence, a suspected vehicle will more often be identified. In the long run, it is also important to strengthen the epidemiological skills of local health authorities so that more detailed epidemiological analyses can be conducted independently.

\section{Acknowledgements}

We would like to thank Susanne Behnke for technical assistance with the epidemic curve spreadsheet, and John Cowden for helpful comments on the manuscript.

\section{Conflict of interest}

None declared.

Authors' contributions

DW has developed the strategic concept for epidemiologic investigation of food-borne disease outbreaks in Germany. HB has developed the analysis tool. Both authors have drafted and revised the manuscript.

\section{References}

1. Directive $2003 / 99 / E C$ of the European Parliament and of the Council of 17 November 2003 on the monitoring of zoonoses and zoonotic agents, amending Council Decision 90/424/ EEC and repealing Council Directive 92/117/EEC. OJ L 325, 12.12.2003, p. 31-40

2. European Food Safety Authority. The European Union summary report on trends and sources of zoonoses, zoonotic agents and food-borne outbreaks in 2010. EFSA Journal. 2012;10:2597(3):442. Available from: http://www.efsa.europa. eu/de/efsajournal/pub/2597.htm

3. Tauxe RV. Emerging foodborne diseases: an evolving public health challenge. Emerg Infect Dis. 1997;3(4):425-34. http:// dx.doi.org/10.3201/eid0304.970403

4. Murphree R, Garman K, Phan Q, Everstine K, Gould LH, Jones TF. Characteristics of foodborne disease outbreak investigations conducted by Foodborne Diseases Active Surveillance Network (FoodNet) sites, 2003-2008. Clin Infect Dis. 2012;54 Suppl 5:S498-503. http://dx.doi.org/10.1093/cid/ cis232

5. Mertens E, Kreher H, Rabsch W, Bornhofen B, Alpers K, Burckhardt F. Severe infections caused by Salmonella 
Enteritidis PT8/7 linked to a private barbecue. Epidemiol Infect. 2012 19:1-7.

6. Gregg MB. Field Epidemiology. First ed. New York: Oxford University Press; 1996.

7. Rothman KJ. Epidemiology: an introduction. New York: Oxford University Press, Inc.; 2002.

8. Launders N, Byrne L, Adams N, Glen K, Jenkins C, TubinDelic D, et al. Outbreak of Shiga toxin-producing E. coli $\mathrm{O}_{157}$ associated with consumption of watercress, United Kingdom, August to September 2013. Euro Surveill. 2013;18(44). pii: 20624.

9. Centers for Disease Control and Prevention (CDC). Outbreak of Salmonella serotype Enteritidis infections associated with raw almonds-United States and Canada, 2003-2004. MMWR Morb Mortal Wkly Rep. 2004;53(22):484-7.

10. Bernard H, Faber M, Wilking H, Haller S, Höhle M, Schielke A, Ducomble T, Siffczyk C, Merbecks SS, Fricke G, Hamouda 0 , Stark K, Werber D, on behalf of the Outbreak Investigation Team. Large multistate outbreak of norovirus gastroenteritis associated with frozen strawberries, Germany, 2012. Euro Surveill. 2014;19(8): pii=20719.

11. Buchholz U, Bernard H, Werber D, Bohmer MM, Remschmidt C, Wilking H, et al. German outbreak of Escherichia coli $0104: \mathrm{H}_{4}$ associated with sprouts. N Engl J Med. 2011;365(19):1763-70. http://dx.doi.org/10.1056/NEJMoa1106482

12. Gormley FJ, Little CL, Rawal N, Gillespie IA, Lebaigue S, Adak GK. A 17-year review of foodborne outbreaks: describing the continuing decline in England and Wales (1992-2008). Epidemiol Infect. 2011;139(5):688-99. http://dx.doi. org/10.1017/So950268810001858

13. Gervelmeyer A, Hempen M, Nebel U, Weber C, Bronzwaer S, Ammon A, et al. Developing the Community reporting system for foodborne outbreaks. Euro Surveill. 2008;13(45): pii: 19029.

14. O'Brien SJ, Gillespie IA, Sivanesan MA, Elson R, Hughes C, Adak GK. Publication bias in foodborne outbreaks of infectious intestinal disease and its implications for evidencebased food policy. England and Wales 1992-2003. Epidemiol Infect. 2006;134(4):667-74. http://dx.doi.org/10.1017/ So950268805005765 